Rabaska

Revue d'ethnologie de l'Amérique française

\title{
Chaire de recherche du Canada en oralité des francophonies minoritaires (Université Sainte-Anne)
}

\section{Jean-Pierre Pichette}

Volume 3, 2005

URI : https://id.erudit.org/iderudit/201742ar

DOI : https://doi.org/10.7202/201742ar

Aller au sommaire du numéro

Éditeur(s)

Société québécoise d'ethnologie

ISSN

1703-7433 (imprimé)

1916-7350 (numérique)

Découvrir la revue

Citer ce document

Pichette, J.-P. (2005). Chaire de recherche du Canada en oralité des

francophonies minoritaires (Université Sainte-Anne). Rabaska, 3, 201-204.

https://doi.org/10.7202/201742ar d'utilisation que vous pouvez consulter en ligne. 


\section{Rapports des institutions}

ACADIE

Chaire de recherche du Canada

en oralité des francophonies minoritaires

Cofram / Centre acadien

Téléphone : (902) 769-2114

Université Sainte-Anne

Télécopieur : (902) 769-0063

Pointe-de-l'Église

(Nouvelle-Écosse) B0W 1M0

Courriel : jeanpierre.pichette@usainteanne.ca

Toile : www.usainteanne.ca

\section{Présentation}

Créée à l'automne 2004, par un généreux octroi du Conseil de recherches en sciences humaines du Canada, la chaire Cofram mène des études comparées sur la littérature orale et les traditions populaires des minorités acadiennes et canadiennes-françaises d'Amérique du Nord. Son programme de recherche examine les processus de diffusion et de rétention des traditions à divers points de vue - ancienneté, origine plurielle, actualité -, et met en rapport les régions périphériques et leur centre. De façon pratique, l'aménagement d'un laboratoire de littérature orale (LABOR) favorisera l'observation de l'Amérique française sous l'angle choisi, l'entraînement d'une équipe d'étudiants et de collaborateurs à l'enquête orale, et le montage d'une réserve documentaire adaptée à la méthode comparative. Situé dans les locaux du Centre acadien $\left(\mathrm{C}_{\mathrm{A}}\right)$, dont le titulaire assume aussi la direction, ce laboratoire profite déjà de la compétence qu'on y a développée depuis sa fondation en 1972 et des collections qui y sont réunies. La chaire, lieu de concertation, de collaboration et d'échanges scientifiques, propose dans ce but une série de manifestations dont elle diffusera les résultats au fur et à mesure.

\section{Démarrage de la chaire}

Le 24 novembre 2004, Monsieur André Roberge, recteur de l'Université Sainte-Anne, présentait officiellement Jean-Pierre Pichette à la communauté de la Baie Sainte-Marie comme le premier détenteur d'une chaire de recherche du Canada dans son institution. Celui-ci livra alors les objectifs et les grands axes des études qu'il entendait poursuivre, marquant du même coup le commencement des activités de la Cofram. Durant le semestre qui a suivi, 
l'attention du titulaire a donc essentiellement été tournée vers des opérations de démarrage : entre autres, l'élaboration des premiers cours de littérature orale, dont l'un fut donné pendant l'hiver 2005, et la mise à jour des investissements de la Fondation canadienne pour l'innovation $(\mathrm{FCl})$ qui commandite l'équipement du laboratoire.

\section{Laboratoire de littérature orale}

Sans tarder, on a saisi dans la banque de données du CA l'inventaire complet de la collection sonore du titulaire et lancé la conversion numérique des 3750 pièces, enregistrées sur bobines et cassettes, tâches préalables à son dépôt dans nos archives. Ainsi, durant tout l'été 2005, une première équipe de quatre assistantes de recherche a pu s'employer aux premiers travaux du laboratoire : dépouillement de la documentation acadienne de la collection Pichette et transcription préliminaire d'environ 225 contes et 350 chansons (NouveauBrunswick et Île-du-Prince-Édouard : 40 heures) ; saisie de 200 contes de la région de Bellechasse (Québec) et de trois recueils d'auteurs régionaux qui ont puisé leurs récits dans la tradition orale. Ces relevés serviront à étayer des études comparatives ou à préparer des éditions critiques. Également, un projet pilote a permis à nos équipes d'entreprendre les premières missions de terrain dans quelques régions acadiennes du sud-ouest de la Nouvelle-Écosse. Ces fonctions répondent aux trois grands volets de recherche de la chaire et en stimuleront la réalisation, notamment en vue de l'établissement d'un corpus de littérature orale de la francophonie nord-américaine (projet ÉCLORE), de la tenue de journées d'études et d'un colloque international sur l'origine plurielle et les identités multiples (projet OpPiDum), tout en consolidant les bases d'une synthèse de nature encyclopédique des traditions populaires à l'usage des ethnologues et chercheurs comme du grand public (projet ENTRAF).

Le 14 avril, $M$. Ronald Labelle, chercheur chevronné du Centre d'études acadiennes et professeur de l'Université de Moncton, inaugurait les conférences de la COFRAM. Son exposé, intitulé « Le Témoignage oral comme marqueur d'identité culturelle en Acadie », mettait à contribution les entrevues qu'il avait menées dans plusieurs communautés acadiennes de la NouvelleÉcosse et éclairait d'un jour nouveau la présence ou l'absence des récits fondateurs.

\section{Poursuite de travaux antérieurs}

Quelques ouvrages collectifs, qui étaient déjà en chantier, ont par ailleurs été menés à bonne fin. Après la publication du deuxième volume de Rabaska, la seule revue d'ethnologie vouée à l'étude des traditions populaires de l'Amérique française, dont le lancement eut lieu à Québec le 10 novembre 2004 (Québec, SQE, 2004, 338 p.), le titulaire a assuré, en collaboration avec 
le comité de rédaction et pour le compte de la Société québécoise d'ethnologie, la coordination du troisième volume. Il ne fait aucun doute que l'ancrage à l'Université Sainte-Anne de cette revue confirme l'axe de la coopération entre les programmes, les centres, les sociétés savantes et les chercheurs de toute l'Amérique française, et conforte aussi les liens établis avec les institutions belges, françaises et étatsuniennes. L'équipe du laboratoire a d'ailleurs accompli diverses tâches intégrées à la production du volume courant de Rabaska, d'autant que la COFRAM en est l'un des partenaires privilégiés.

Le titulaire a supervisé la publication du sixième Cahier Charlevoix, organe de la Société Charlevoix dont il est le rédacteur et l'un des auteurs (Sudbury, Prise de parole, 2004, 295 p.). De plus, par l'édition du livre important de Jean Simard, Le Québec pour terrain. Itinéraire d'un missionnaire du patrimoine religieux, il a relancé la collection des «Archives de folklore » fondée en 1946 par Luc Lacourcière (Québec, PUL, «AF » 28, 2004, [10]-242 p.).

\section{Organisation du congrès de l'ACEF}

Grâce au personnel de la Cofram et du $\mathrm{C}_{\mathrm{A}}$, l'Université Sainte-Anne a pu accueillir le vingt-neuvième congrès annuel de l'Association canadienne d'ethnologie et de folklore (ACEF). Exceptionnellement, l'ACEF s'est détachée du congrès des sciences humaines et sociales pour souligner le $400^{\mathrm{e}}$ anniversaire de l'établissement de Port-Royal en 1605. Durant les trois jours du congrès, du 18 au 22 mai 2005 , plus de cinquante communications ont été offertes aux membres de l'association qui représentaient une douzaine de centres universitaires et musées des Maritimes, du Québec, de l'Ontario et de l'Ouest canadien, ainsi que des États-Unis. Pour l'ethnologie, la rencontre fut aussi mémorable par la participation des titulaires des quatre grandes chaires de recherche à une table ronde, où chacun put résumer ses travaux et orientations : Beverley Diamond, chaire en ethnomusicologie (Memorial University, obtenue en juillet 2002) ; Laurier Turgeon, chaire sur les patrimoines ethnologiques (Université Laval, juin 2003) ; Jean-Pierre Pichette, chaire CofRAM (Université Sainte-Anne, juillet 2004) ; et Richard MacKinnon, chaire sur le patrimoine immatériel (Cape Breton University, octobre 2004). $\mathrm{Au}$ cours de cette réunion, l'ACEF a remis le Prix Marius-Barbeau 2005 à l'ethnologue Jean Simard, professeur retraité de l'Université Laval, auteur d'une dizaine d'ouvrages consacrés au patrimoine religieux.

\section{Ordre des francophones d'Amérique}

À Québec, le 15 mars 2005, le Conseil supérieur de la langue française du Québec accueillait dans l'Ordre des francophones d'Amérique Jean-Pierre 
Pichette et reconnaissait le travail qu'il avait jusque-là accompli pour l'avancement de l'ethnologie en Ontario français, où il avait œuvré de 1981 à 2004 au département de Folklore et ethnologie de l'Université de Sudbury. C'est la ministre de la Culture et des communications qui lui remit les insignes de l'ordre au cours d'une cérémonie qui se déroula au salon rouge de l'Assemblée nationale.

JeAn-Pierre Pichette 